\title{
Estructura, ontogenia y vascularización de las flores e inflorescencias de Drimys granadensis (Winteraceae)
}

\author{
Xavier Marquínez \\ Departamento de Biología, Facultad de Ciencias, Universidad Nacional de Colombia. Bogotá, Colombia; \\ xmarquinezc@unal.edu.co
}

\author{
Recibido 12-VIII-2013. Corregido 10-XII-2013. Aceptado 28-I-2014.
}

\begin{abstract}
Structure, ontogeny and vascularization of the flowers and inflorescences of Drimys granadensis (Winteraceae). Drimys granadensis is a widespread species in montane forests of South and Central America. In this research, the structure, ontogeny, phyllotaxis and vascularization of the flowers and inflorescences of this species was studied in a population from the Eastern hills of Sabana de Bogota, Colombia. The methods used applied both optical microscopy, with astra blue-fuchsin staining, and scanning electron microscopy, using critical point dryed and gold-paladium metallized samples. Besides, results were compared with those of Drimys winteri, a widely studied species distributed in Chile and Argentina. Additionally, we studied the detail of the floral anatomy to determine the bracteal or calicine identity of the caliptra. I confirmed the proliferative status of the monothelic inflorescence, discarding alternative explanations of the terminal flower identity. I found that uniflorescences have an acropetal development until the terminal meristem becomes the terminal flower, then this flower develops rapidly resulting in a determined uniflorescence. I found pseudosyphonosthelic vascularization in peduncles and pedicels. Besides, I discovered some evidence in the vascular and anatomical structures, to consider the caliptra as the fusion product of various structures and therefore of calicine origin. The caliptra showed a whorled phyllotaxis, but the petals, stamens and carpels presented a spiral condition; phyllotaxis change was explained by the long time lapse between the initiation of the calyx and the corolla. I found great similarities among the inflorescences of $D$. granadensis and $D$. winteri; they were different in the proliferation start time, and in the frequent presence of nomophylls in $D$. granadensis, in contrast to the presence of reduced bracts and bracteoles in D. winteri inflorescences. Rev. Biol. Trop. 62 (2): 743-756. Epub 2014 June 01.
\end{abstract}

Key words: calyptra, monothelic inflorescence, phyllotaxis, proliferation, uniflorescence, vascularization.

Winteraceae ha sido considerada durante mucho tiempo como una familia de diversificación temprana en las angiospermas (Takhtajan, 1980; Cronquist, 1981) conformada por cinco géneros; Takhtajania y Zygogynum s.l. con crecimiento simpodial debido a la presencia de inflorescencias terminales (Vink, 1993) y Drimys, Pseudowintera y Tasmannia con crecimiento monopodial, debido a la proliferación del eje inflorescencial en los dos primeros géneros (Weberling, 1988); o a la presencia de inflorescencias caulifloras en Tasmannia (Sampson, 1963; Vink, 1970).

Históricamente se han utilizado caracteres asociados a la inflorescencia, tales como posición (axilar o "terminal") y número de flores de la inflorescencia parcial, en la taxonomía del género Drimys (Linnaeus f., 1781; Miers, 1858); sin embargo Smith (1943b), en su revisión del género, señaló que estos caracteres son inconstantes y deben ser utilizados con precaución. Trabajos posteriores han estudiado varios aspectos de las inflorescencias de Drimys winteri J. R. Forst. \& G. Forst.: vascularización (Nast, 1944), estructura (Weberling, 1988), ontogenia de flores e inflorescencias (Tucker, 1959; Vink, 1970; Doust, 2001).

En Winteraceae la flor esta conformada por una caliptra externa conformada por la unión congénita de un verticilo de estructuras 
foliáceas, las cuales han sido interpretadas como de origen calicino (Smith, 1943a; Smith,1943b; Nast, 1944; Bailey \& Nast, 1945; Hiepko, 1965; Tucker \& Gifford, 1966; Sampson, Williams, \& Woodland, 1988; Vink, 1993; Endress, 1996; Doust, 2000; Endress, Igersheim, Sampson, \& Schatz, 2000; Doust \& Drinnan, 2004); o de origen de bracteal (Endress et al., 2000; Doust \& Drinnan, 2004); seguida de pétalos (o tépalos), estambres y carpelos siguiendo una filotaxis verticilada. La interpretación que se haga de la caliptra afecta las hipótesis de homología relacionadas con las brácteas inflorescenciales y con el perianto de la flor en Winteraceae y en relación con su familia hermana, las Canellaceae.

Marquínez, Lohmann, Faria-Salatino, Salatino, \& González (2009), basados en análisis de secuencias nucleares (ITS) y de cloroplasto ( $r p \mathrm{~S} 16$ y $p s b \mathrm{~A}-t r n \mathrm{H})$ propusieron la existencia de dos clados en Drimys; uno de distribución suroccidental $\left(>30^{\circ}\right.$ de latitud sur) conformado por Drimys confertifolia Phil., D. winteri y $D$. Andina (Reiche) Rodr. \& Quez. y el otro nororiental (entre $30^{\circ}$ latitud sur $-23^{\circ}$ latitud norte) constituido por $D$. angustifolia Miers, D. brasiliensis Miers, D. granadensis L.f. y D. roraimensis (A.C.Sm.) Ehrend. \& Gottsb. Dado que los estudios previos de estructura, ontogenia y vascularización de las flores e inflorescencias se han centrado en la especie $D$. winteri J.R. Forst. \& G. Forst. (Chile y Argentina), correspondiente al clado suroccidental, este trabajo pretende estudiar estos mismos aspectos en $D$. granadensis, especie de amplia distribución (Bosques montanos centroamericanos y bosques andinos de Colombia, Ecuador y Perú) en el clado nororiental, para poder establecer similitudes y diferencias; busca además evidencias que permitan definir la naturaleza bracteal o calicina de la caliptra de Winteraceae.

\section{MATERIALES Y MÉTODOS}

Inflorescencias de $D$. granadensis en diferentes estado de desarrollo pertenecientes a una población situada en Altos de Yerbabuena, cerros orientales de la Sabana de Bogotá
(452'40" N - 7400'04" W; 2808 m.s.n.m.; Cundinamarca, Colombia. Ejemplares testigo: Marquínez 001-004, COL), fueron colectados y fijados en FAA (10:5:85, formol: acido acético glacial: EtOH 70\%) por 24 horas, luego transferidas a EtOH $70 \%$ y tratadas con los protocolos convencionales modificados de Johansen (1940) así: Deshidratación en series de EtOH (70 al 100\%) y de EtOH 100\%-histoclear $(90: 10,70: 30,50: 50,30: 70,10: 90,100)$ cada una por 12 horas; imbibición en parafina $\left(60^{\circ} \mathrm{C}\right)$, tres cambios por 24 horas c/u; secciones seriadas o individuales en micrótomo de rotación y fijación en láminas con ayuda de adhesivo de Haupt; desparafinado mediante pasos de Xilol, Xilol:EtOH 100\%, EtOH 100\%, $95 \%, 70 \%, 50 \%$; tinción en safranina por $18 \mathrm{hr}$, lavado en agua destilada y deshidratación en EtOH 50\%, 70\% y 95\%; contratinción con fast-green por 12 minutos, deshidratación en EtOH 100\%, EtOH70:Xilol 30, EtOH 50:xilol 50 , xilol $100 \%$, colocación de una gota de citoresina y cubreobjetos. Los micropreparados fueron observados con un microscopio Nikon Eclipse E-200 y para las observaciones bajo estereoscopio se empleó un equipo Nikon SMZ 800; en ambos casos se tomaron las fotos con una cámara Nikon 950 Coolpix incorporada a los equipos ópticos.

Para microscopía electrónica de barrido, las muestras fijadas en FAA y EtOH 70\% fueron disectadas, secadas por su punto crítico en un equipo Bal-tec CPB030, metalizadas con oro-paladio en un metalizador FisonsPolaron, modelo SC-500, y examinadas y fotografiadas en un microscopio electrónico de barrido Quanta 200 FEI del Laboratorio de Microscopía Electrónica, Centro de Equipos Interfacultades de la Universidad Nacional de Colombia (CEIF).

La estructura general de la inflorescencia fue revisada en cerca de 406 ejemplares de $D$. granadensis y 129 de $D$. winteri, provenientes de 20 herbarios (Marquínez, 2009). Con el fin de unificar terminología, en esta investigación los términos inflorescencia y uniflorescencia son utilizados en el sentido de Doust (2001; Fig. 1A); por lo tanto, inflorescencia es 


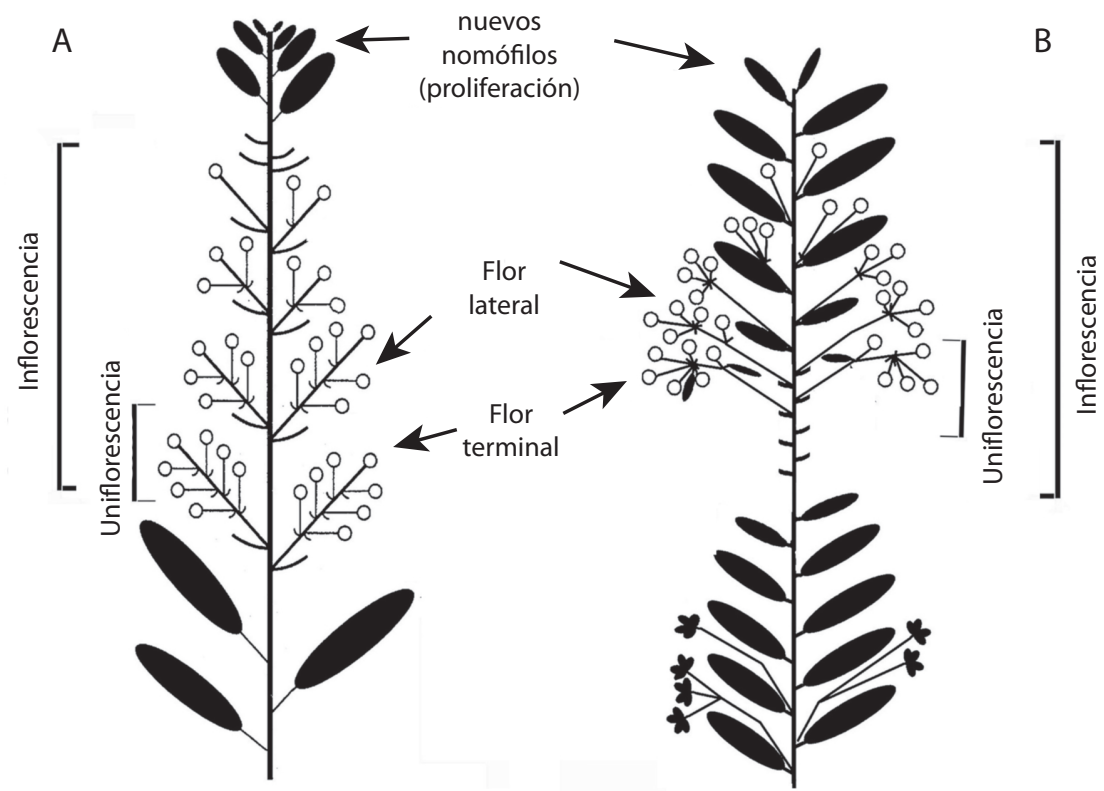

Fig. 1. Representaciones esquemáticas de inflorescencias de (A.) D. winteri (modificada de Doust 2001) y (B.) D. granadensis.

Fig. 1. Schematic representations of (A.) D. winteri inflorescence (modified of Doust 2001) and (B.) D. granadensis inflorescence.

equivalente a rama inflorescencial (sensu Tucker, 1959) y uniflorescencia equivale a inflorescencia sensu Nast (1944) y Tucker (1959) o a paracladio sensu Weberling (1988).

\section{RESULTADOS}

\section{Estructura y filotaxis de la inflorescen-} cia: La inflorescencia de D. granadensis (Fig. 1B) presenta un eje principal proliferante, esto es, un eje principal que no finaliza en una flor terminal, sino que reanuda el crecimiento vegetativo; por tanto las uniflorescencias siempre son laterales y con una conformación umbelada con un número variable de entre 2-13 flores.

En algunos casos la uniflorescencia puede tener una o dos flores a diferente nivel por debajo de la umbela (botrioide). En cualquier caso, la base del pedicelo es articulada y se encuentra acompañada generalmente por brácteas que se desprenden rápidamente, o por nomófilos persistentes (Fig. 1B); la flor terminal de cada uniflorescencia carece de bráctea (Fig. 2 A, Fig. 2 B). En los ejemplares de herbario de $D$. granadensis revisados ocasionalmente se observa una clara alternancia de fases de desarrollo vegetativas y reproductivas, con flores e inflorescencias axilares a brácteas y bracteolas; sin embargo, lo más frecuente es que las uniflorescencias y flores estén localizadas en la axila de nomófilos.

La disposición de las flores en las uniflorescencias parece alterna (Fig. 2B); no obstante, los entrenudos se acortan y el ángulo formado entre flores consecutivas varía a tal grado que puede llegar a tener una disposición decusada (Fig. 2A). Esto contrasta fuertemente con la regularidad filotáctica en el ángulo de dispersión de $138.46^{\circ}$ de las hojas y yemas alternos en los ápices vegetativos, y de las brácteas y uniflorescencias en los ápices reproductivos.

Ontogenia de inflorescencias y flores: El meristema apical de las ramas floríferas de D. granadensis (Fig. 3A) es aplanado. 
A
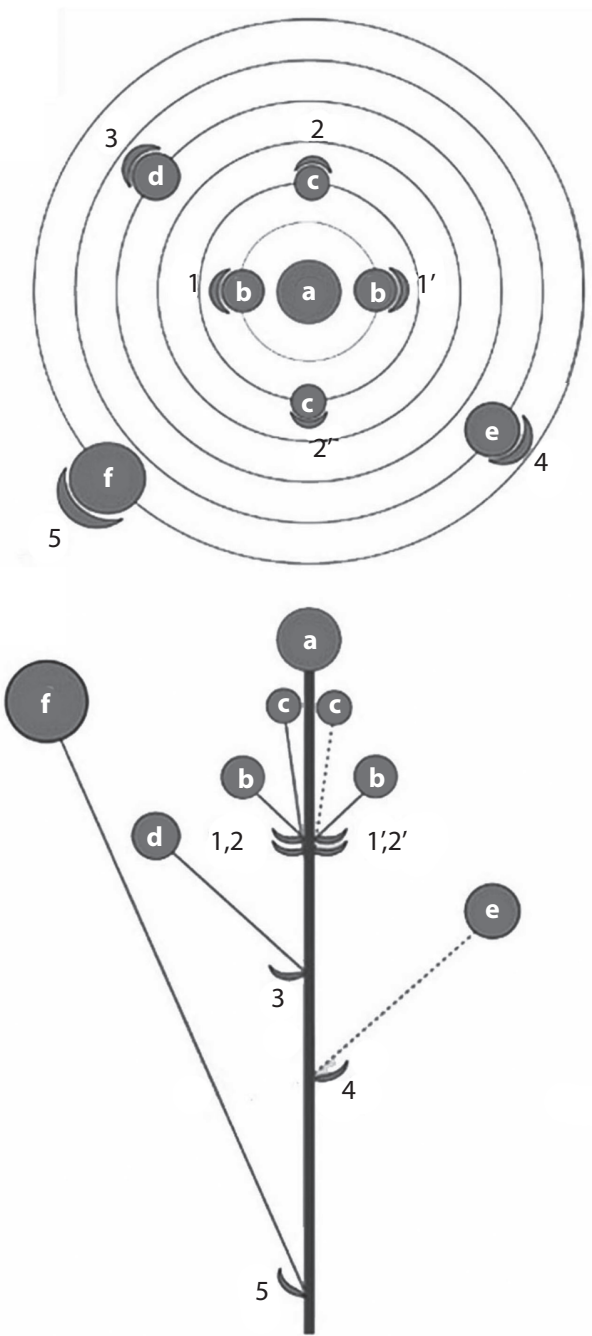
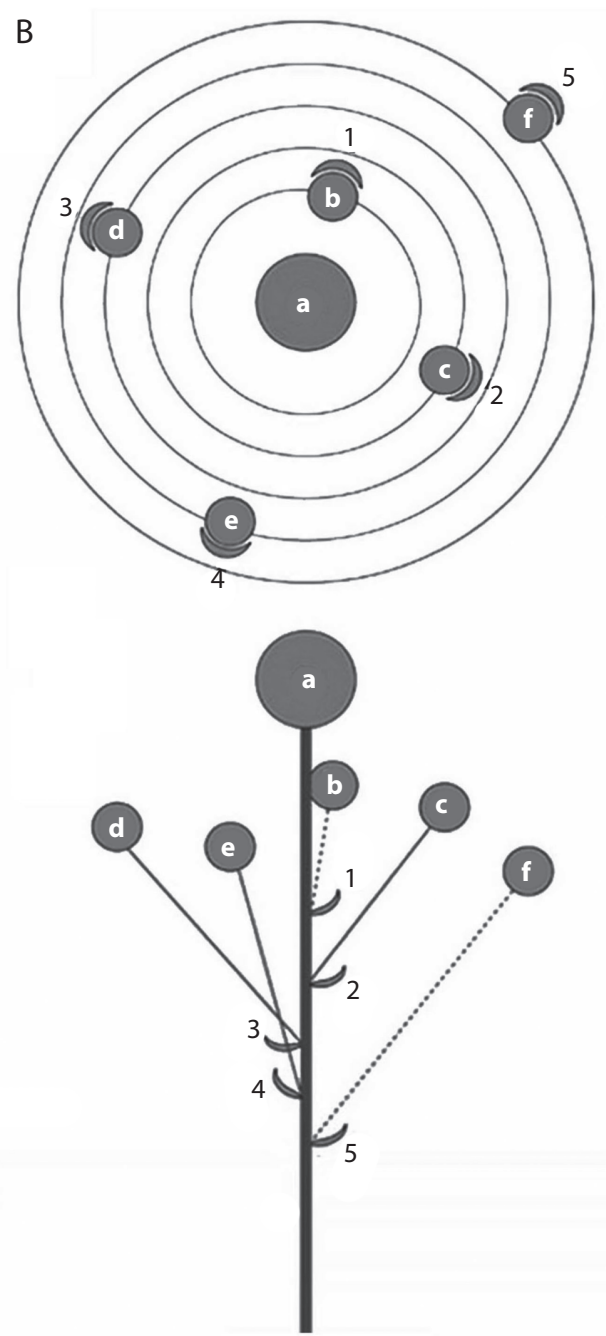

Fig. 2. Representaciones esquemáticas de dos uniflorescencias de D. granadensis en vista superior y lateral, basadas en cortes seriados de uniflorescencias muy jóvenes. Las flores se han señalado con letras (a para la flor terminal), el diámetro es proporcional al grado de desarrollo del botón floral; las bracteolas se señalan con números.

Fig. 2. Schematic representations of two uniflorescences of $D$. granadensis in top and side view, based on very young uniflorescence serial sections. The flowers are marked with letters (a to the terminal flower), the diameter is proportional to the degree of flower bud development; the bracteoles are indicated by numbers.

Los primordios foliares se originan en arreglo helicoidal. En el tercer o cuarto primordio de bráctea, se forma axilarmente la primera yema uniflorescencial (Fig. 3B) como un área lenticular de células meristemáticas separadas del parénquima vacuolado cercano por un arco de varias capas célulares en división activa llamada la "zona escutelar" ("shell zone" sensu
Tucker, 1959). La zona escutelar tiene un mayor número de capas en el lado que da hacia el eje del ápice vegetativo (Fig. 3B) y las divisiones iniciales son periclinales con referencia a la superficie de la yema axilar correspondiente. Las capas más externas de la zona escutelar conforman una túnica de dos capas (Fig. 3B, Fig. 3C), en tanto que el cuerpo principal da 

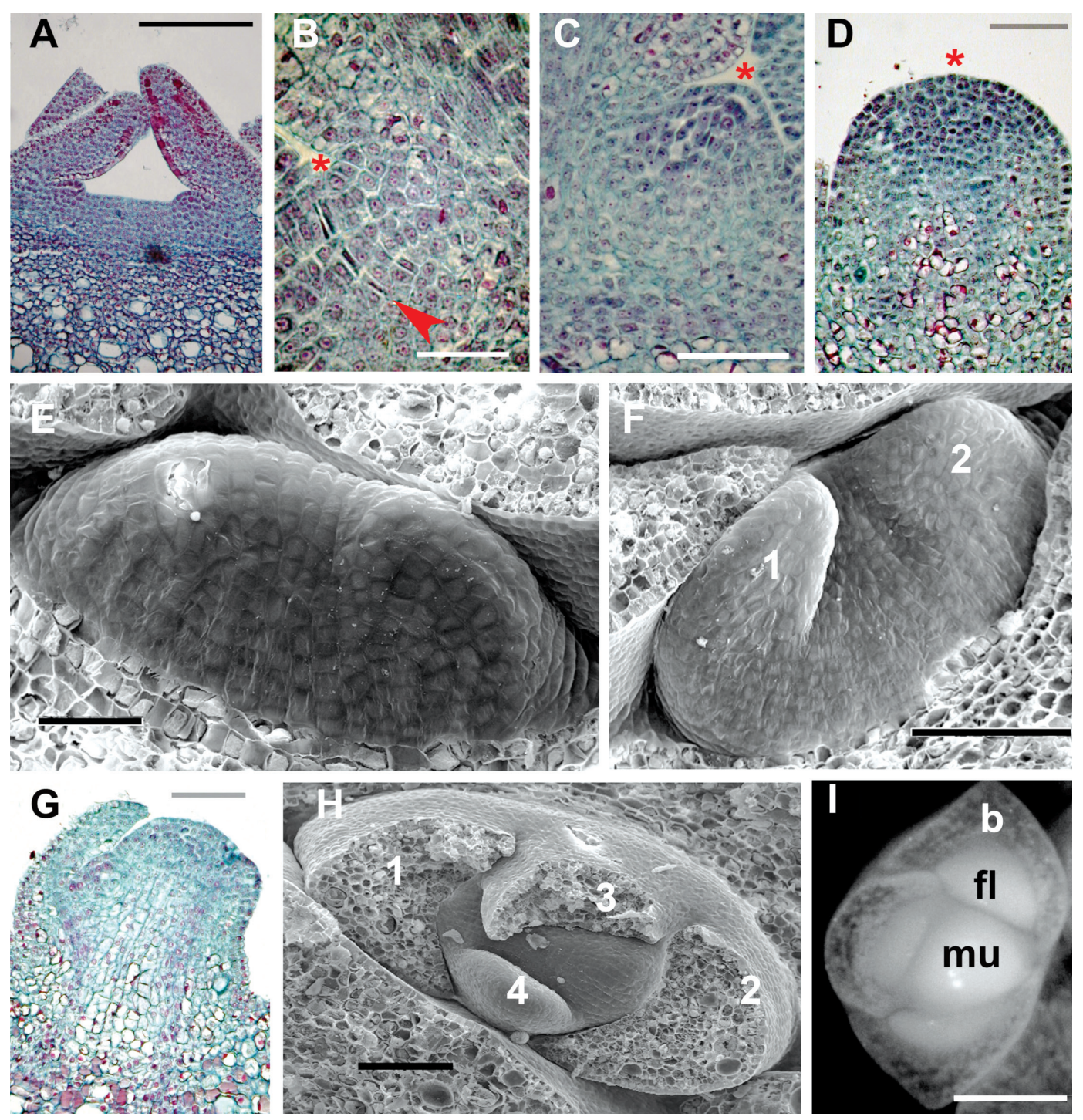

Fig. 3. Ontogenia de la inflorescencia de D. granadensis. A. Ápice de la inflorescencia, sección longitudinal; B.-D. fases sucesivas de desarrollo de la yema uniflorescencial; los asteriscos indican la protodermis de la yema uniflorescencial y la flecha señala la zona escutelar; E. Yema uniflorescencial indiferenciada; F. Yema uniflorescencial desarrollando las dos primeras bracteolas (1-2), G., H. Yema inflorescencial con cuatro bracteolas (1-4); I. uniflorescencia con tres primordios florales laterales (fl) y meristema uniflorescencial (mu) sin diferenciar aún en flor terminal, las tres bracteolas (b) se han removido; A.-E., G.,=microscopio óptico, E.-F., H.=microscopia electrónica de barrido, I.=Estereomicroscopio; Escalas: 50 um en B., C., E.; 100 um en D., F.-H.; $200 u m$ en A., I.

Fig. 3. Ontogeny of the inflorescence of D. granadensis. A. Inflorescence apex, longitudinal section; B.-D. Successive stages of uniflorescencial bud development; the asterisks indicate the protodermis of uniflorescencial bud and the arrow points to the shell zone; E. indiferenciated uniflorescencial bud, F. Uniflorescencial bud developing the two first bracteoles $(1,2)$, G., H. inflorescencial bud with four bracteoles (1-4); I. uniflorescence with three lateral flower primordia (ft) and uniflorescencial meristem $(\mathrm{mu})$ without differentiating in terminal flower, the three bracteolas (b) have been removed A-E.,G.=optic microscopy, E.-F., H.=scanning electronic microscopy; I=Estereomicroscopy. Scales: 50 $\mu$ m in B., C., E.; $100 \mu \mathrm{m}$ in D., F.-H.; $200 \mu \mathrm{m}$ in A., I. 
lugar al corpus; la división de la segunda capa de la túnica y del corpus da lugar a un crecimiento convexo de la yema uniflorescencial (Fig. 3C).

La yema uniflorescencial incrementa en tamaño y adquiere una configuración globosa (Fig. 3D), hasta la conformación de las primeras dos bractéolas, las cuales se forman en los flancos laterales y disposición aparentemente opuesta dándole una apariencia elipsoidal (Fig. $3 \mathrm{E}$, Fig. 3F); posteriormente se forman la tercera y cuarta bractéolas en posición medial (Fig. 3G, Fig. 3H); durante la transición de dos a cuatro bractéolas, la túnica y el corpus dan lugar a la diferenciación de los meristemas primarios (protodermis, meristema fundamental y procámbium; Fig. 3G). Las brácteas crecen rápidamente en tanto que, en posición axilar y dirección acropétala se originan los primordios florales axilares (Fig. 3I, 4A); sin embargo, cuando el meristema apical de la uniflorescencia se diferencia en flor terminal (Fig. 4B), las flores laterales interrumpen su desarrollo hasta que la flor terminal ha conformado totalmente la caliptra y ha iniciado la formación de pétalos; por ello, la flor terminal se ve rápidamente como la más desarrollada de la uniflorescencia (Fig. 4B). En las uniflorescencias jóvenes, la detención del crecimiento debida a la flor terminal ocurre principalmente en aquellas flores localizadas en la parte umbelada de la inflorescencia; las flores formadas por debajo de la umbela no sufren dicha inhibición y pueden presentar un mayor grado de desarrollo que la flor terminal (Fig. 2A; la flor $\mathrm{f}$ presenta mayor desarrollo que la a, terminal).

El desarrollo de las flores laterales de $D$. granadensis comienza con un primordio de contorno elipsoidal (Figs. 3I, 4A); cuando el primordio floral es de aproximadamente $260 \mu \mathrm{m}$ de diámetro se forman dos primordios laterales (Fig. 4C, Fig. 4D, Fig. 4E, Fig. 4F) que son empujados hacia arriba por la formación de un anillo caliptral de contorno elipsoidal (Fig. 4B), el cual crece hasta cubrir el ápice floral indiferenciado; cuando el primordio floral supera los $430 \mu \mathrm{m}$ de diámetro, los primeros pétalos se inician en posición lateral (Fig. 4F) y continúan su iniciación en sentido acrópeto y disposición helicoidal; seguido de los estambres y carpelos.

El meristema apical vegetativo de la uniflorescencia (Fig. 3I) se convierte en el primordio aproximadamente globoso y ebracteado de la flor terminal; cuando el diámetro de este primordio es de aproximadamente $200 \mu \mathrm{m}$ se forma un meristema anular periférico que da lugar a la caliptra que bordea el ápice floral (Fig. 4B, Fig. 4C, Fig. 4D, Fig. 4E, Fig. 4F, Fig. $4 \mathrm{G})$; la caliptra crece hasta cubrir totalmente el ápice floral que permanece indiferenciado (Fig. $4 \mathrm{H}$ ); cuando el primordio alcanza un diámetro mayor a $450 \mu \mathrm{m}$ comienza la diferenciación en sentido acrópeto y disposición helicoidal de los pétalos (Fig. 4I) seguido por los estambres y carpelos (Fig. 4J, Fig. 4K).

Vascularización de la inflorescencia: En los pedúnculos y pedicelos de D. granadensis, el desarrollo temprano de cámbium interfascicular produce rápidamente un cilindro ininterrumpido de células derivadas que determina la conformación de una pseudosifonostela (sensu Sauquet et al., 2003; Figs. 5A); hacia el interior de dicho anillo, de manera discontinua, se dispone el protoxilema ya diferenciado. En cada uno de los nudos de las uniflorescencias, la pseudosifonostela se fracciona dando lugar a tres trazas nodales y tres trazas foliares que irrigan las bractéolas (Fig. 5B, Fig. 5C, Fig. 5D, Fig. 5 E, Fig. 5F); estas trazas foliares se intercalan con dos trazas que divergen para vascularizar el pedicelo de la flor correspondiente (Fig. 5C, Fig. 5D, Fig. 5E, Fig. 5F, Fig. 5G), en forma de arco; posteriormente las dos trazas se fusionan formando de nuevo una pseudosifonostela en el pedicelo de cada flor lateral (Fig. 5D, Fig. 5E, Fig. 5F, Fig. 5G). La flor terminal carece de bracteola y no existe ningún vestigio vascular de ésta (Fig. 5F, Fig. 5G, Fig. 5H, Fig. 5I, Fig. 5J); el pedicelo de la flor terminal es irrigado por un número variable de trazas remanentes (entre dos y cuatro; cf. Fig. 5G, con tres trazas remanentes) que quedan luego de la conformación de las flores subterminales 

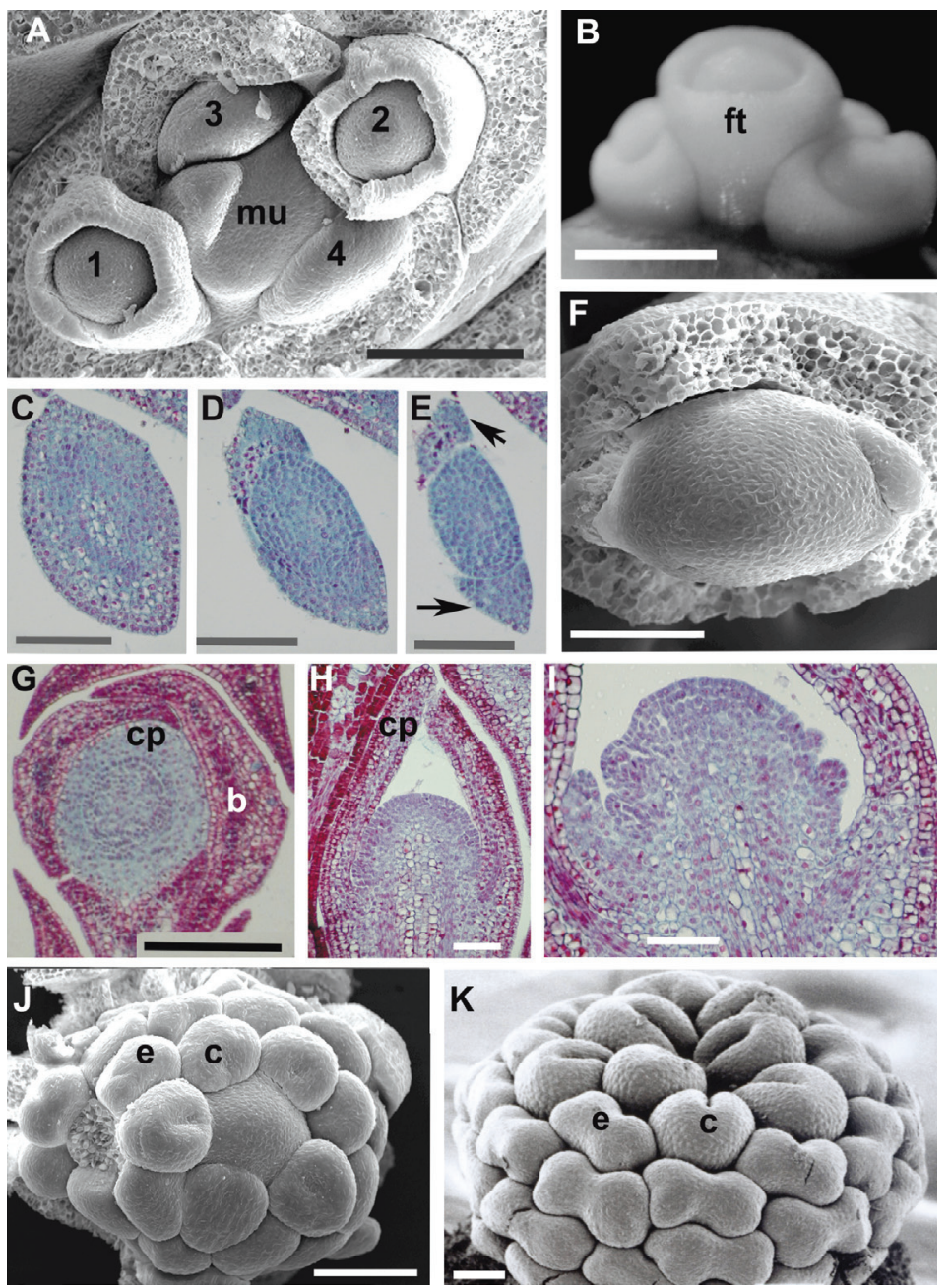

Fig. 4. Ontogenia de la uniflorescencia (A., B.), de las flores laterales (C.-F.) y terminales (G.-K.) de D. granadensis A. Uniflorescencia con desarrollo acrópeto, los primordios florales más basales $(1,2)$ con caliptra anular, los siguientes primordios $(3,4)$ inician los lóbulos caliptrales laterales, meristemo uniflorescencial indiferenciado (mu); B. Uniflorescencia con flor terminal caliptrada (ft) superando en desarrollo a las tres flores laterales; C.-E. Secciones transversales seriales de flor lateral, nótese la presencia de dos lóbulos caliptrales laterales (flechas); F. Flor lateral (caliptra retirada), nótese la presencia de dos pétalos en posición lateral; G. Flor terminal con caliptra anular (cp); tres bracteolas (b) de flores laterales la rodean; H. Sección longitudinal de flor terminal, la caliptra (cp) cubre el ápice floral antes de comenzar la diferenciación de pétalos; I. Flor terminal en sección longitudinal, inicio de pétalos y estambres; J.-K. flor terminal con primordios de pétalos, estambres (e) y carpelos (c), la caliptra ha sido removida. A., F. ,J. , K.=microscopio electrónico de barrido,

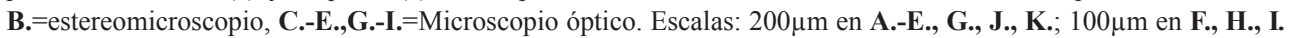

Fig. 4. Ontogeny of uniflorescence (A,B), of the lateral (CF) and terminal flowers (GK) of D. granadensis A. Uniflorescence with acropetal development, most basal flower primordia (1.2) with ringshaped caliptra, the following primordia (3.4) start lateral caliptral lobes, undifferentiated uniflorescencial meristem (mu); B. Uniflorescence with caliptrated terminal flower (ft) surpassing in development the three lateral flowers, C.-E. Serial cross sections of lateral flower, note the presence of two lateral caliptral lobes (arrows); F. Lateral flower (calyptra removed), note the presence of two petals in lateral position; G. Terminal flower with ringshaped caliptra (cp), three bracteoles (b) of lateral flowers surrounds, H. Longitudinal section of terminal flower, the caliptra (cp) covers the floral apex before the differentiation of petals; I. Terminal flower in longitudinal section beginning development of petals and stamens, J.-K. Terminal flower with primordia of petals, stamens (e) and carpels (c), the caliptra has been removed. A., F., J., K.=Scanning electronic microscope, B.=stereomicroscope, C.-E., G.-I.=optical microscope. Scales: $200 \mu \mathrm{m}$ in A.-E., G. , J., K.; $100 \mu \mathrm{m}$ in F., H. ,I. 

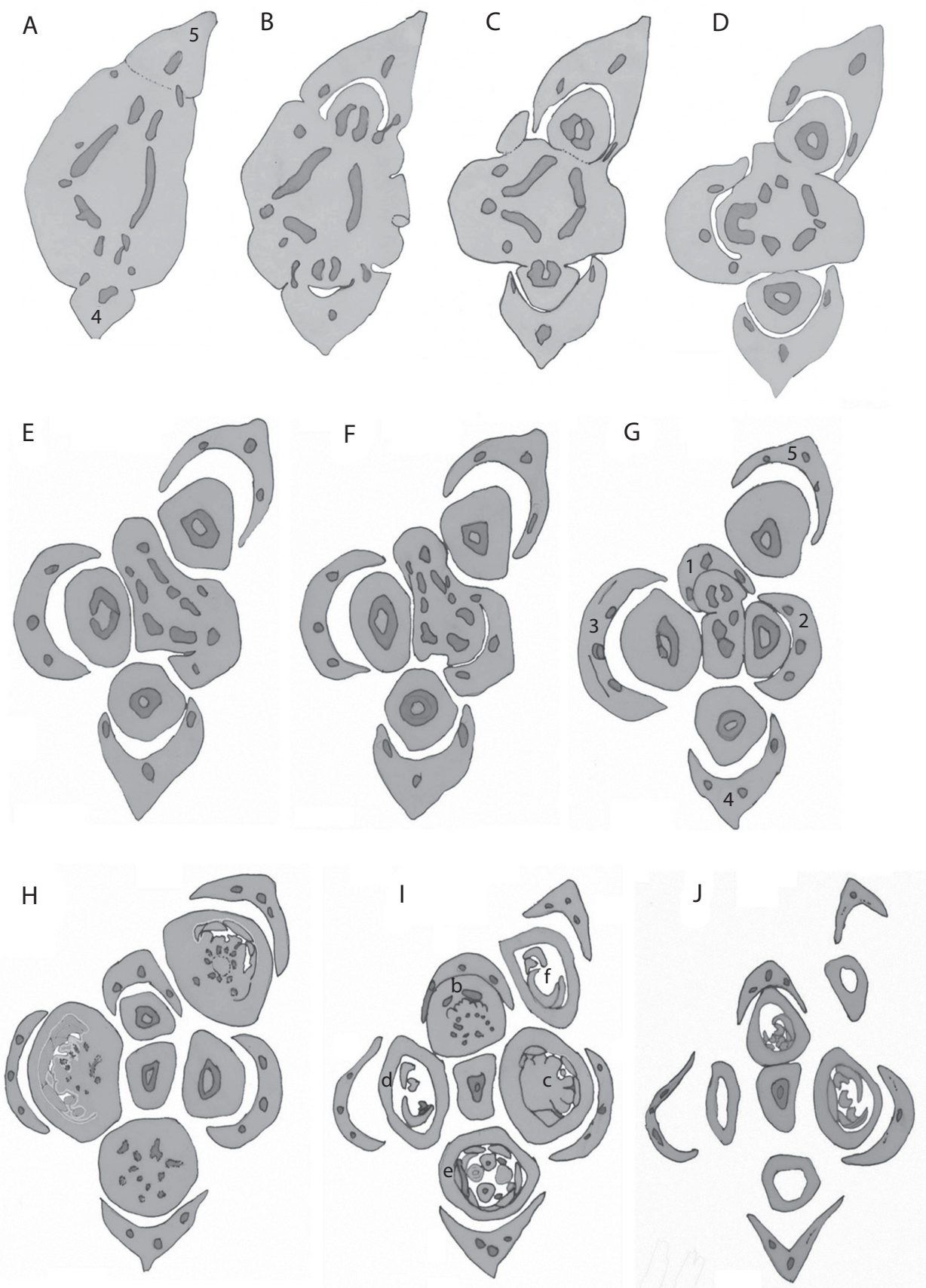

Fig. 5. Serie de secciones transversales de una inflorescencia joven de D. granadensis, desde la base de la inflorescencia (A) hasta la conformación del pedicelo de la flor terminal (J). La brácteas están numeradas (1-5) y las flores señaladas con letras minusculas (b-f); manteniendo las convenciones de la Fig. 2A que representa esta misma inflorescencia en vista superior y lateral. En gris oscuro se indican los haces vasculares.

Fig. 5. Series of cross sections of a young inflorescence of $D$. granadensis, from the base of the inflorescence (A) until the formation of the terminal flower stalk $(\mathrm{J})$. The bracts are numbered (1-5) and flowers indicated with lowercase letters (b-f) maintaining the conventions of Fig. 2A representing the same inflorescence in top and side view. Vascular bundles are indicated in dark grey. 
y que reconstituyen la pseudosifonostela en su pedicelo (Fig. 5J).

Vascularización y simetría floral: La caliptra de las flores laterales de D. granadensis se origina de un primordio disimétrico elipsoidal con dos lóbulos laterales (Fig. 4B, Fig. 4C, Fig. 4D); alcanzando siempre un nivel más alto en los lados, seguido de la zona adaxial (Fig. 4 A, Fig. B); en las flores laterales los primeros haces de la caliptra se desprenden de la pseudosifonostela (Fig. 6A, Fig. B, Fig. C) proveniente del pedicelo en posición abaxial; posteriormente otros haces inervan los flancos laterales (Fig. 6D) y finalmente otros haces llegan a la región adaxial de la caliptra (Fig. 6E, Fig. 6E F, Fig. 6E G). En total, cinco a siete haces vasculares divergen de la pseudosifonostela a diferentes niveles; éstos a su vez se dividen sucesivamente en dos o tres haces cada vez, de tal manera que la caliptra totalmente formada presenta 18-22 haces hacia la parte media de la flor (Fig. 6G). La vascularización confiere a la caliptra de la flor lateral una simetría monosimétrica (Fig. 6A, Fig. 6B, Fig. 6C, Fig. 6D, Fig. 6E, Fig. 6F, Fig. 6G).

En las flores terminales, la caliptra se forma como un anillo a partir de un primordio redondeado (Fig. 4G, Fig. 4H); está vascularizada por seis a siete haces que no divergen simultáneamente (Fig. 6H, Fig. 6I, Fig. 6J, Fig. 6K, Fig. 6L, Fig. 6M, Fig. 6N, Fig. 6O, Fig. 6P, Fig. 6Q) y que se ramifican en 17-23 haces hacia la parte media de la flor. La vascularización confiere a la caliptra una simetría monosimétrica, aunque menos marcada que en las flores laterales.

\section{DISCUSIÓN}

En Drimys las inflorescencias han sido tradicionalmente consideradas como monotélicas proliferantes (Weberling, 1988) o monotélicas truncadas (Weberling, 1992). La condición proliferante o truncada implica que el eje principal del sistema inflorescencial culmina en un meristema que luego de la floración retoma el crecimiento vegetativo. Las fases vegetativa y reproductiva son muy claras en $D$. winteri, donde las inflorescencias y flores se presentan axilares a brácteas y la proliferación es tardía; mientras que en $D$. granadensis las flores e inflorescencias frecuentemente se presentan axilares a nomófilos y la activación del meristemo terminal de la inflorescencia ocurre cuando aún se están desarrollando las flores.

La condición monotélica implica la presencia de flor terminal en las uniflorescencias, las cuales corresponderían a racimos determinados que adquiere la forma de umbelas por empobrecimiento de los paracladios y acortamiento de los entrenudos (botrioide sensu troll; cf. Weberling, 1992); este último concepto explicaría la presencia de flores individuales por debajo de la umbela terminal, en una zona donde los entrenudos no se han acortado.

En estudios ontogénicos, Doust (2001) determina que en $D$. winteri los primeros primordios florales se presentan en posición lateral al meristemo terminal indiferenciado y subopuestas una a la otra; la iniciación de brácteas y primordios florales se da en dirección acrópeta antes de que el meristema uniflorescencial eventualmente se transforme en flor terminal; cuando ocurre este evento, la flor terminal se desarrolla rápidamente. Por tanto, las uniflorescencias son determinadas pero su desarrollo es acrópeto; en $D$. granadensis el desarrollo sigue el mismo modelo.

La presencia en D. granadensis de uniflorescencias con flores terminales diferentes a las laterales (por ser ebracteadas y más simétricas), sumado a su desarrollo acrópeto podría tener una explicación alternativa a la propuesta por Weberling (1988; 1992): Sokoloff, Rudall, \& Remizowa (2006) proponen tres posibles tipos de estructura terminal en las inflorescencias: (a) una verdadera flor terminal, (b) un pseudanto por unión de flores laterales y (c) una flor lateral que ha cambiado a posición pseudoterminal. En Drimys, varios criterios señalados por Sokoloff et al. (2006) apuntan a que se presenta una verdadera flor terminal: (1) aunque su origen es tardío (desarrollo acrópeto), sufre antesis antes que las flores laterales superiores, (2) carece de bractea (varias brácteas indicarían 

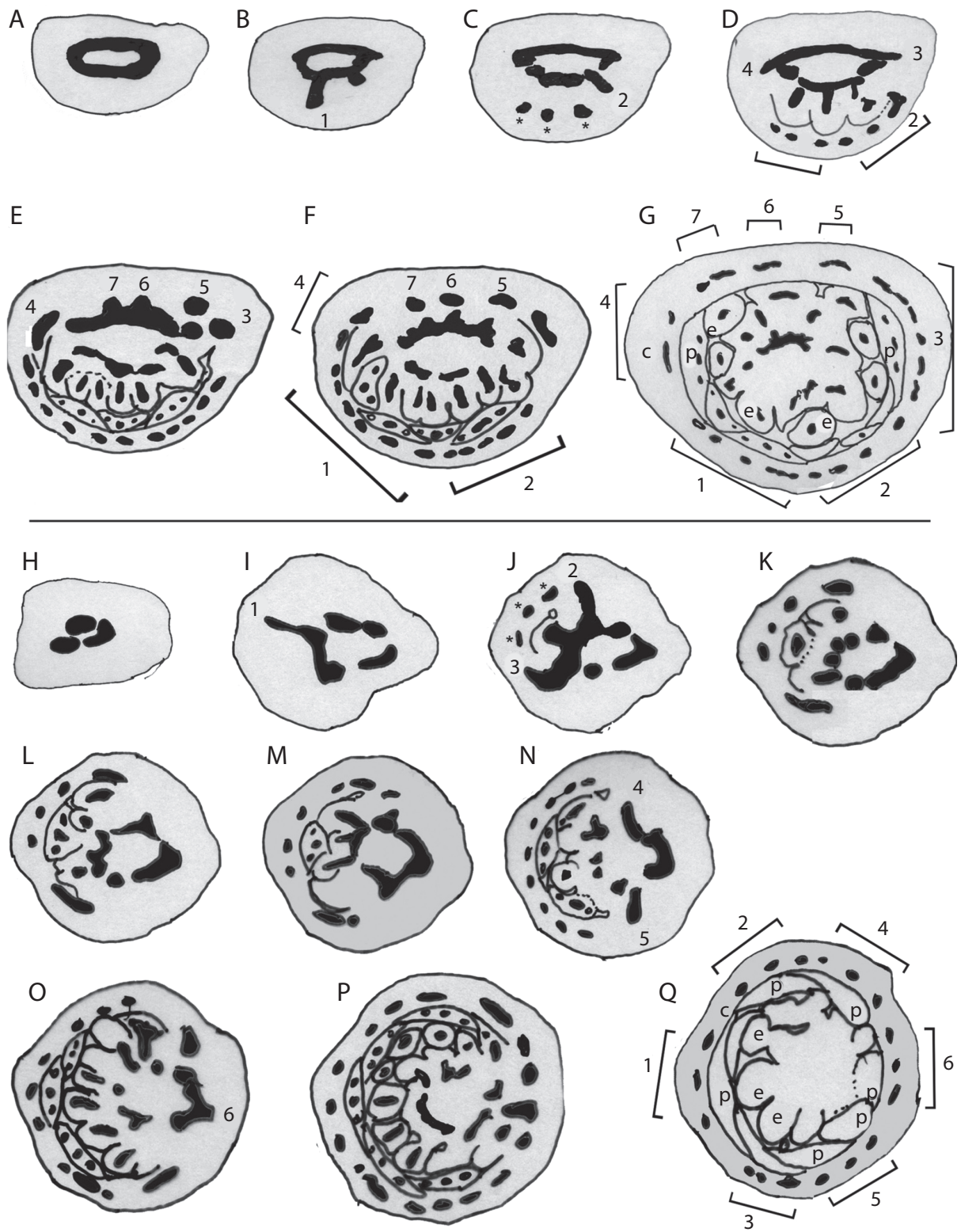

Fig. 6. Representación esquemática de secciones transversales de una flor lateral (A.-G.) y una flor terminal (H.-Q.) de $D$. granadensis; respectivamente desde el pedúnculo (A. o H.), hasta la parte media del botón floral (G. o Q.). En B. e I. se numera el primer haz vascular primario (1) que diverge de la pseudosifonostela y en $\mathrm{C}$ y J los haces secundarios derivados de éste se señalan con asteriscos. Se numeran los haces vasculares primarios (1-7 en la flor lateral, 1-6 en la flor terminal) y en G. y Q. se indica con corchetes la vascularización derivada de cada haz primario. En $\mathbf{Q}$. se ha omitido la vascularización de pétalos (p) y estambres (e).

Fig. 6. Schematic representation of cross sections of a lateral flower (A.-G.) and a terminal flower (H.-Q.) of D. granadensis, respectively from the stem (A. or H.) until the middle of the flower bud (G. or Q.). In B. and I. numbered the first primary vascular bundle (1) that diverges from the pseudosifonostela and in $\mathbf{C}$. and $\mathbf{J}$. the secundary bundles derived from it are indicated by asterisks. Primary vascular bundles are numbered (1-7 in lateral flower, 1-6 in terminal flower) and in G. and Q. the vascularization derived from each primary vascular bundle is indicated by brackets. In Q the vascularization of petals (p) and stamens (e) is ommited. 
pseudanto, una bractea indicaría flor pseudoterminal), (3) residuo apical ausente en la uniflorescencia, (4) simetría monosimétrica en todas las flores, al presentar el cáliz monosimétrico y los verticilos florales internos (pétalos, estambres y carpelos) asimétricos debido a su inserción espiralada, (5) vascularización floral similar por pseudosifonostela derivada de dos haces en flor lateral y dos a cuatro en flor terminal. En conclusión, la evidencia favorece la interpretación tradicional de inflorescencias monotélicas proliferantes para $D$. granadensis.

La distribución de los haces vasculares a lo largo de los pedúnculos y pedicelos de Winteraceae fue interpretado inicialmente como una eustela (Nast 1944); sin embargo nuestro trabajo indica que los pedúnculos y pedicelos de D. granadensis presentan pseudosifonostelas, condición compartida por Takhtajania (Keating, 2000) y Canellaceae (Wilson, 1965; Wilson, 1966), con lo cual se propone como una posible sinapomorfía para el orden Canellales.

Se han propuesto dos hipótesis acerca de la identidad de la caliptra en Winteraceae: bracteal o calicina; adicionalmente, e independiente de su origen, puede ser también producto de un solo órgano o fusión de varios órganos. Para determinar si la caliptra de Winteraceae se origina a partir de brácteas o sépalos es necesario realizar una distinción entre estos dos órganos; definiciones tentativas propuestas por Endress (2003) señalan que los sépalos (así como los pétalos y los tépalos) son órganos integrados en la organización de la flor, en tanto que las brácteas son simples filomas que no se han diferenciado en nomófilos y que no están integrados en la flor en su estado maduro; siguiendo este criterio, la caliptra de Winteraceae sería de origen calicino puesto que se forma solamente luego de que el ápice vegetativo se ha diferenciado en ápice floral y está integrada en la flor en su estado maduro.

Existen dos aproximaciones para intentar resolver el problema de la naturaleza de la caliptra en Winteraceae; (1) indirectamente buscando estructuras similares a la caliptra en grupos filogenéticos próximos y (2) directamente mediante el estudio de desarrollo y anatomía.
En cuanto a la comparación con grupos filogenéticos próximos, en Eumagnoliales se presenta fusión de sépalos únicamente en Aristolochiaceae (González \& Stevenson, 2000a); en Zygogynum p.p., Canella y Cinnamosma se presenta fusión de pétalos (Wilson, 1966; Doust, 2000); por el contrario, brácteas simples que conforman caliptras se presentan en grupos menos relacionados filogenéticamente, como algunas Annonaceae que presentan una yema floral encerrada por algún tiempo por la bráctea precedente sobre el pedicelo (Endress, 1977) o en Galbulimima (Himantandraceae) con dos caliptras (una externa y otra interna) correspondientes a dos brácteas tubulares dispuestas consecutivamente (Endress, 1977; Endress, 2003) o en Eupomatia (Eupomatiaceae) con caliptra bracteal amplexicaule (Endress, 2003; Kim et al., 2002). No existen caliptras formadas por varias brácteas en Eumagnoliales, pero sí en Sarcandra y Chloranthus (Chloranthaceae; von Balthazar \& Endress, 1999). En conclusión, la filogenia no aporta evidencia conclusiva sobre el origen de la caliptra en Winteraceae.

En relación con el desarrollo y anatomía de la caliptra, mientras que en Eupomatia la caliptra bracteal está precedida por brácteas amplexicaules con las que existe una continuidad filotáctica y la vascularización indica que la caliptra es un órgano simple (Endress, 2003), en Winteraceae el anillo meristemático no precedido por lóbulos caliptrales en las flores terminales de $D$. granadensis y $D$. winteri es la única evidencia que apoya la idea de que la caliptra pueda derivar de un órgano único (bráctea, sépalo o pétalo); sin embargo, la confluencia de la vascularización hace pensar en la fusión de dos o tres órganos cada uno con tres trazas (Nast, 1944) y la presencia de dos primordios laterales al comienzo del desarrollo del anillo caliptral en flores laterales indican que es un órgano dímero; en tanto que las brácteas, bracteolas y nomófilos de Winteraceae se presentan siempre de manera solitaria en cada nudo. En cambio, una caliptra dímera calicina presenta continuidad merística con la corola dímera a tetrámera en otras Winteraceae (Marquínez, 2009). Teniendo en cuenta todo lo 
anterior, la evidencia aquí presentada sustenta, aunque no de manera conclusiva, que la caliptra de Winteraceae es de origen calicino.

La condición plesiomórfica para el número de sépalos es de tres en Canellales y Piperales, (Ronse de Craene, Soltis, \& Soltis, 2003); ésta se presenta en Canellaceae (Hammel \& Zamora, 2005), y en Aristolochiaceae (González \& Stevenson, 2000b); el cáliz dímero (eventualmente tetrámero o indeterminado) resulta ser una sinapomorfía para las Winteraceae (Marquínez, 2009). Los dos sépalos que forman la caliptra de las flores laterales de las Winteraceae se originan en posición lateral en Drimys, Pseudowintera, Takhtajania, Zygogynum s.l., o en posición medial en Tasmannia, siendo ésta una sinapomorfía para las especies de este último género (cf. Doust, 2000; Doust \& Drinnan, 2004). La caliptra de las flores terminales de $D$. granadensis (esta investigación) y de $D$. winteri (Doust, 2001) es indeterminado en cuanto al número de partes y posición relativa de las mismas porque se origina de un meristema completamente anular.

La disposición de pétalos, estambres y carpelos en Drimys se ha descrito como espiralada (Baillon, 1868), verticilada (Smith, 1943b), irregular (Hiepko, 1965), alterada (Erbar \& Leins, 1983) o más o menos desordenada (Endress, 1996). Doust (2001) encontró en $D$. winteri una gran variabilidad filotáctica. En $D$. winteri (Doust, 2001) y D. granadensis (esta investigación), el cambio de filotaxis de una caliptra calicina aparentemente verticilada a una disposición espiralada de pétalos, estambres y carpelos durante el desarrollo floral se ha relacionado con el tiempo prolongado entre el inicio de la formación del cáliz y el inicio de formación de los primeros pétalos, con lo cual se pierde la continuidad filotáctica que normalmente daría lugar a un patrón decusado de los pétalos externos en relación con los sépalos (cf. Doust, 2000; Doust, 2001; Doust \& Drinnan, 2004). Este cambio, verticilado en sépalos a espiralado en pétalos, es una sinapomorfía del género Drimys, dado que la posición de los pétalos externos es alterna en relación con los sépalos en Pseudowintera,
Takhtajania, Tasmannia, Zygogynum amplexicaule, Z. comptonii, Z. crassifolium, y Z. pancheri; mientras que es opuesta en $Z$. baillonii, Z. bicolor, Z. pomiferum y Z. stipitatum. La condición opuesta de los primeros sépalos respecto a los pétalos es una sinapomorfía para $Z$. sensu Baillon (Doust, 2000; Doust, 2001). En Canellaceae, familia hermana de Winteraceae, tanto el cáliz como la corola son verticilados, pero frecuentemente con un número diferente de partes (Hammel \& Zamora, 2005), así que su relación posicional es también indefinida, excepto en Cinnamosma donde es alterna (Baillon, 1868).

En conclusión, D. granadensis y D. winteri presentan una gran similaridad en la estructura, ontogenia, filotaxis y vascularización de flores e inflorescencias. Se presentan diferencias puesto que en $D$. winteri las uniflorescencias y flores se presentan axilares a brácteas y la proliferación es tardía, mientras que en $D$. granadensis las flores e inflorescencias frecuentemente son axilares a nomófilos y la proliferación temprana; esto es, cuando las flores aún no han entrado en antesis. En $D$. granadensis la vascularización de los ejes de la inflorescencia y de los pedicelos es pseudosifonostélica, condición que se propone como sinapomorfía para el orden Canellales. Toda la evidencia apunta a que la caliptra de Drimys es de origen calicino, con filotaxis verticilada, cambiando a filotaxis helicoidal de pétalos, estambres y carpelos. Sin embargo, la identidad de la caliptra deberá ser confirmada en un futuro con estudios de biología del desarrollo similares a los desarrollados en Eupomatia (Kim et al., 2002).

\section{AGRADECIMIENTOS}

A Favio González por sus valiosos comentarios y orientaciones en la elaboración del escrito y en la investigación, al Instituto para el Desarrollo de la Ciencias y la Tecnología "Francisco José de Caldas", a COLCIENCIAS por el préstamo-beca mediante el Programa de apoyo a doctorados nacionales 2004 


\section{y a la Universidad Nacional de Colombia por la contrapartida.}

\section{RESUMEN}

Drimys granadensis es una especie de amplia distribución en los bosques montanos de Sur y Centro América. En esta investigación se estudiaron, mediante microscopía óptica y electrónica de barrido, la estructura, ontogenia, filotaxis y vascularización de sus flores e inflorescencias, y fueron comparadas con las de Drimys winteri, especie distribuida en Chile y Argentina. Adicionalmente, se buscó evidencia para determinar la identidad bracteal o calicina de la caliptra de sus flores. Se confirmó la condición monotélica proliferante de la inflorescencia, descartando explicaciones alternativas de identidad de la flor terminal. Las uniflorescencias presentan un desarrollo acrópeto, hasta que el meristemo terminal se transforma en flor terminal, entonces esta flor se desarrolla rápidamente dando lugar a una uniflorescencia determinada. La vascularización es pseudosifonostélica para pedúnculos y pedicelos. Se encontró evidencia en la vascularización y estructura anatómica para considerar la caliptra como el producto de fusión de varias estructuras y, por tanto, de origen calicino. La caliptra presentó una filotaxis verticilada, pero los pétalos, estambres y carpelos presentaron una filotaxis espiralada; el cambio se explicó por el tiempo prolongado entre la iniciación de cáliz y corola. Las inflorescencias de $D$. granadensis y $D$. winteri son muy similares; siendo diferente el tiempo de inicio de la proliferación y la frecuente presencia de nomófilos en las inflorescencias de $D$. granadensis, en contraste con la presencia de brácteas y bracteolas reducidas en $D$. winteri.

Palabras clave: caliptra, filotaxis, inflorescencia monotelica, proliferación, uniflorescencia, vascularización.

\section{REFERENCIAS}

Bailey, I. W. \& Nast, C. G. (1945). The comparative morphology of the Winteraceae. VII. Summary and conclusions. Journal of Arnold Arboretum, 26, 37-47.

Baillon, H. (1868). Historie des Plantes (Magnoliacées). Librairie de L. París: Hachette et Cie.

Balthazar, M. von. \& Endress, P. K. (1999). Floral bract function, flowering process and breeding systems of Sarcandra and Chloranthus (Chloranthaceae). Plant Systematics and Evolution, 218, 161-178.

Cronquist, A. (1981). An Integrated System of Classification of Flowering Plants. New York: Columbia University Press.

Doust, A. N. (2000). Comparative floral ontogeny in Winteraceae. Annals of the Missouri Botanical Garden, 87, 366-379.
Doust, A. N. (2001). The developmental basis of floral variation in Drimys winteri (Winteraceae). International Journal of Plant Science, 162, 697-717.

Doust, A. N. \& Drinnan, A. N. (2004). Floral development and molecular phylogeny support the generic status of Tasmannia (Winteraceae). American Journal of Botany, 91, 321-331.

Endress, P. K. (1977). Über blütenbau und verwandtschaft der Eupomatiaceae und Himantandraceae (Magnoliales). Berichte der Deutschen Botanischen Gesellschaft, 90, 83-103.

Endress, P. K. (1996). Diversity and Evolutionary Biology of Tropical Flowers. Cambridge: Cambridge University Press.

Endress, P. K. (2003). Early floral development and nature of the calyptra in Eupomatiaceae (Magnoliales). International Journal of Plant Science, 164, 489-503.

Endress, P. K., Igersheim, A., Sampson, F. B., \& Schatz, G. E. (2000). Floral structure of Takhtajania perrieri and its systematic position in Winteraceae. Annals of the Missouri Botanical Garden, 87, 347-365.

Erbar, C. \& Leins, P. (1983). Zur Sequenz von Blutenorganen bei einigen Magnoliiden. (On the sequence of floral organs in some Magnoliidae.) Botanische Jahrbücher für Systematik, 103, 433-449.

González, F. \& Stevenson, D. W. (2000a). Gynostemium development in Aristolochia (Aristolochiaceae). Botanische Jahrbücher für Systematik, 122, 249-291.

González, F. \& Stevenson, D. W. (2000b). Perianth development and systematics of Aristolochia. Flora, 195, 370-391

Hammel, B. E. \& Zamora, N. E. (2005). Pleodendron costaricense (Canellaceae), a new species for Costa Rica. Lankesteriana, 5, 211-218.

Hiepko, P. (1965). Vergleichend-morphologische und entwicklungsgeschichtliche untersuchungen über das perianth bei den polycarpicae. Botanische Jahrbücher für Systematik, 84, 359-508.

Johansen, D. J. (1940). Plant Microtechnique. New York: McGraw-Hill Book.

Keating, R. C. (2000). Anatomy of the young vegetative shoot of Takhtajania perrieri (Winteraceae). Annals of the Missouri Botanical Garden, 87, 335-346.

Kim, S., Soltis, D. E., Soltis, P. S., Endress, P. K., Hauser, B. A., \& Zanis, M. J. (2002). Origin of the calyptra and characterization of B-class genes in Eupomatia bennettii (Eupomatiaceae). International Journal of Plant Science, 166, 185-198.

Linnaeus, F. C. (1781). Supplementum Plantarum Systematis Vegetabilium, editionis decimae tertiae, Generum plantarum editionis sextae, et Specierum 
plantarum editionis secunda. Brunsvigae: Impenfis Orphanotrophei.

Marquínez, X., Lohmann, L. G., Faria-Salatino, M. L., Salatino, A., \& González, F. (2009). Generic relationships and dating lineages in Winteraceae based on nuclear (ITS) and plastid ( $r p \mathrm{~S} 16$ and $p s b \mathrm{~A}-t r n \mathrm{H})$ sequence data. Molecular Phylogenetics and Evolution, 53, 435-449.

Marquínez, X. (2009). Filogenia y biogeografía del género Drimys (Winteraceae). (Tesis de Doctorado). Universidad Nacional de Colombia, Bogotá, Colombia.

Miers, J. (1858). On the Winteraceae. Annals and Magazine of Natural History. Third Series, 2, 33-48, 109-115.

Nast, C. G. (1944). The comparative morphology of Winteraceae. VI. Vascular anatomy of the flower shoot. Journal of Arnold Arboretum, 26, 37-47.

Ronse de Craene, L. P., Soltis, P. S., \& Soltis, D. E. (2003). Evolution of floral structures in basal angiosperms. International Journal of Plant Science, 164 (5 Suppl.): S329-S363.

Sampson, F. B. (1963). The floral morphology of Pseudowintera, the New Zealand member of the vesselless Winteraceae. Phytomorphology, 13, 403-423.

Sampson, F. B., Williams, J. B., \& Woodland, P. S. (1988). The morphology and taxonomic position of Tasmannia glaucifolia (Winteraceae), a new Australian species. Australian Journal of Botany, 36, 395-413.

Sauquet, H., Doyle, J. A., Scharaschkin, T., Borsch, T., Hilu, K. W., Chatrow, L. W., \& Le Thomas, A. (2003). Phylogenetic analysis of Magnoliales and Myristicaceae based on multiple data sets: implications for character evolution. Botanical Journal of the Linnean Society, 142, 125-186.
Smith, A. C. (1943a). Taxonomic notes on the old world species of Winteraceae. Journal of the Arnold Arboretum, 24, 119-164.

Smith, A. C. (1943b). The American species of Drimys. Journal of the Arnold Arboretum, 24, 1-33.

Sokoloff, D., Rudall, P., \& Remizowa, M. (2006). Flowerlike terminal structures in racemose inflorescences: a tool in morphogenetic and evolutionary research. Journal of Experimental Botany, 57, 3517-3530.

Takhtajan, A. L. (1980). Outline of the Classification of Flowering Plants (Magnoliophyta). Botanical Review, 46, 277-299.

Tucker, S. C. (1959). Ontogeny of the inflorescence and flower in Drimys winteri var. chilensis. University of California Publications in Botany, 30, 257-336.

Tucker, S. \& Gifford, E. M. (1966). Organogenesis in the carpellate flower of Drimys lanceolata. American Journal of Botany, 53, 433-442.

Vink, W. (1970). The Winteraceae of the Old World I. Pseudowintera and Drimys - Morphology and taxonomy. Blumea, 18, 226-354.

Vink, W. (1993). Winteraceae. In K. Kubitzki, J. G. Rohwer, \& V. Bittich (Eds.), The families and genera of vascular plants, vol. II (pp. 630-638). Berlín: Springer-Verlag.

Weberling, F. (1988). Inflorescence structure in primitive angiosperms. Taxon, 37, 657-690.

Weberling, F. (1992). Morphology of flowers and inflorescences. Cambridge: Cambridge University Press.

Wilson, T. K. (1965). The comparative morphology of the Canellaceae. II. Anatomy of the young stem and node. American Journal of Botany, 52, 369-378.

Wilson, T. K. (1966). The comparative morphology of the Canellaceae. IV. Floral morphology and conclusions. American Journal of Botany, 53, 336-343. 